19 December 2001

\title{
Integration of a generalized Hénon-Heiles Hamiltonian
}

\author{
C. Verhoeven†, M. Musette†, and R. Conte* \\ $\nmid$ Dienst Theoretische Natuurkunde, Vrije Universiteit Brussel \\ Pleinlaan 2, B-1050 Brussels, Belgium \\ E-mail: cverhoev@vub.ac.be and mmusette@vub.ac.be \\ * Service de physique de l'état condensé, CEA-Saclay \\ F-91191 Gif-sur-Yvette Cedex, France \\ E-mail: Conte@drecam.saclay.cea.fr
}

PACS: 45.20.Jj-Lagrangian and Hamiltonian mechanics; 05.45.Yv-Solitons

\begin{abstract}
The generalized Hénon-Heiles Hamiltonian $H=1 / 2\left(P_{X}^{2}+P_{Y}^{2}+c_{1} X^{2}+\right.$ $\left.c_{2} Y^{2}\right)+a X Y^{2}-b X^{3} / 3$ with an additional nonpolynomial term $\mu Y^{-2}$ is known to be Liouville integrable for three sets of values of $\left(b / a, c_{1}, c_{2}\right)$. It has been previously integrated by genus two theta functions only in one of these cases. Defining the separating variables of the Hamilton-Jacobi equations, we succeed here, in the two other cases, to integrate the equations of motion with hyperelliptic functions.
\end{abstract}




\section{Introduction}

The generalization of the Hénon-Heiles Hamiltonian defined by

$$
\begin{aligned}
H & \equiv \frac{1}{2}\left(P_{X}^{2}+P_{Y}^{2}+c_{1} X^{2}+c_{2} Y^{2}\right)+a X Y^{2}-\frac{b}{3} X^{3}+\frac{1}{32 a^{2}} \frac{\mu}{Y^{2}}, a \neq 0 \\
X^{\prime} & =P_{X}, Y^{\prime}=P_{Y} \\
X^{\prime \prime} & =-a Y^{2}+b X^{2}-c_{1} X \\
Y^{\prime \prime} & =-2 a X Y-c_{2} Y+\frac{1}{16 a^{2}} \frac{\mu}{Y^{3}}, \mu \text { arbitrary }
\end{aligned}
$$

is integrable in the sense of Liouville in three cases, 13 namely

$$
\begin{aligned}
& \frac{b}{a}=-1, \quad c_{1}=c_{2}, \\
& \frac{b}{a}=-6, \quad c_{1}, c_{2} \text { arbitrary, } \\
& \frac{b}{a}=-16, \quad c_{1}=16 c_{2},
\end{aligned}
$$

and is equivalent to the reduction $\xi=x-c t$ of three fifth order soliton equations, 1 respectively the Sawada-Kotera (SK), $\mathrm{KdV}_{5}$ and Kaup-Kupershmidt (KK) equations, by applying the translation

$$
\begin{aligned}
\mathrm{SK}: & u=X+\frac{c_{2}}{2 a}, \quad c=c_{1} c_{2}, \\
\mathrm{KdV}_{5}: & u=X+\frac{c_{1}+4 c_{2}}{20 a}, \quad c=\frac{1}{10}\left(-3 c_{1}^{2}-16 c_{1} c_{2}+48 c_{2}^{2}\right), \\
\mathrm{KK}: & u=X+\frac{c_{2}}{2 a}, \quad c=c_{1} c_{2} .
\end{aligned}
$$

The reduced partial differential equations (PDE's) are respectively

$$
\begin{aligned}
\mathrm{SK}: & u^{(4)}+10 a u u^{\prime \prime}+\frac{20}{3} a^{2} u^{3}-c u+4 a E-\frac{c_{1} c_{2}^{2}}{3 a}=0 \\
\mathrm{KdV}_{5}: & u^{(4)}+20 a u u^{\prime \prime}+40 a^{2} u^{3}+10 a u^{\prime 2}-c u \\
& +4 a E+\frac{1}{100 a}\left(c_{1}+4 c_{2}\right)\left(c_{1}^{2}-12 c_{1} c_{2}+16 c_{2}^{2}\right)=0 \\
\mathrm{KK}: & u^{(4)}+40 a u u^{\prime \prime}+\frac{320}{3} a^{2} u^{3}+30 a u^{\prime 2}-c u+4 a E-\frac{c_{1} c_{2}^{2}}{3 a}=0
\end{aligned}
$$

in which $E$ is the constant value of the Hamiltonian $H$. Therefore we will further use these names for refering to the respective integrable cases of the Hamiltonian. 
The problem which we address is to find the separating variables and to explicitly integrate.

The general solution for the $\mathrm{KdV}_{5}$ case has been obtained in terms of hyperelliptic functions 1 by separation of the variables of the HamiltonJacobi equation in parabolic coordinates.

For $\mu \overline{\bar{B}} 0$ the equations of motion for the SK and KK cases have been integrated 10 in terms of elliptic functions.

In this paper we give the general solution of the equations of motion for the SK and KK cases in terms of hyperelliptic functions. This is achieved by separation of the variables of the Hamilton-Jacobi equation, where the canonical transformation between SK and KK⿴囗1 plays an important role.

In section II, we consider the SK case with $\mu=0$ as a starting point to treat the general case ( $\mu$ arbitrary). In section [II], we recover the canonical transformation between SK and KK, starting from the factorization of the scattering operator associated with these equations and using the link with the Fordy-Gibbons equation.12 In section [V], we give the general solution for $\mathrm{KK}$, using the canonical transformation previously obtained. In section $\mathrm{V}$, we use the fact that the canonical transformation is invertible for obtaining the general solution of the SKcase. In both cases, in the limit $\mu \rightarrow 0$, we recover the previous results. 10 In section $\mathrm{VI}$, we compare our method of integration, starting from the Hamiltonian system, with the method used by Cosgrove 13 for integrating the fourth order ODE's (11) and (13).

\section{Particular case: Sawada-Kotera for $\mu=0$}

This is the simplest case and it will be our starting point to define the separation of variables of the Hamilton-Jacobi equation for KK.

Consider the Hamiltonian in the SK case derived from (11) by setting $a=\frac{1}{2}$ and defining $, U=X+c_{2}, V=Y, c=c_{1} c_{2}$

$$
H \equiv K_{1,0}=\frac{1}{2}\left(P_{U}^{2}+P_{V}^{2}\right)+\frac{1}{2} U V^{2}+\frac{1}{6} U^{3}-\frac{c}{2} U,
$$

which is a constant of motion of the equations

$$
\begin{aligned}
& U^{\prime \prime}=-\frac{1}{2}\left(V^{2}+U^{2}\right)+\frac{c}{2}, \\
& V^{\prime \prime}=-U V,
\end{aligned}
$$

where $U, V$ and the derivatives $U^{\prime}=P_{U}, V^{\prime}=P_{V}$ are functions of the independent variable $\xi\left({ }^{\prime} \equiv \frac{\mathrm{d}}{\mathrm{d} \xi}\right)$. 
This system possesses a second constant of motion 9

$$
K_{2,0}=-2 P_{U} P_{V}-U^{2} V-\frac{V^{3}}{3}+c V
$$

which is in involution with the Hamiltonian, i.e. $\left\{K_{1,0}, K_{2,0}\right\}=0$.

The separation of variables is defined as

$$
\begin{array}{ll}
Q_{1}=U+V, & Q_{2}=U-V, \\
P_{1}=\frac{1}{2}\left(P_{U}+P_{V}\right), & P_{2}=\frac{1}{2}\left(P_{U}-P_{V}\right),
\end{array}
$$

and in those new variables the expressions of $K_{1,0}$ and $K_{2,0}$ are

$$
\begin{aligned}
& K_{1,0}=P_{1}^{2}+P_{2}^{2}+\frac{1}{12}\left(Q_{1}^{3}+Q_{2}^{3}\right)-\frac{c}{4}\left(Q_{1}+Q_{2}\right), \\
& K_{2,0}=2\left(P_{2}^{2}-P_{1}^{2}\right)+\frac{1}{6}\left(Q_{2}^{3}-Q_{1}^{3}\right)-\frac{c}{2}\left(Q_{2}-Q_{1}\right),
\end{aligned}
$$

such that the equations of motion become

$$
\left\{\begin{array}{l}
Q_{1}^{\prime 2}=-\frac{1}{3} Q_{1}^{3}+c Q_{1}-K_{2,0}+2 K_{1,0} \\
Q_{2}^{\prime 2}=-\frac{1}{3} Q_{2}^{3}+c Q_{2}+K_{2,0}+2 K_{1,0}
\end{array}\right.
$$

They possess the general solution

$$
\left\{\begin{array}{l}
Q_{1}=-12 \wp\left(\xi-\xi_{1}, \frac{c}{12},-\frac{1}{144}\left(2 K_{1,0}-K_{2,0}\right)\right) \equiv-12 \wp_{1}(\xi), \\
Q_{2}=-12 \wp\left(\xi-\xi_{2}, \frac{c}{12},-\frac{1}{144}\left(2 K_{1,0}+K_{2,0}\right)\right) \equiv-12 \wp_{2}(\xi),
\end{array}\right.
$$

in terms of the Weierstrass elliptic function $\wp\left(x-x_{0}, g_{2}, g_{3}\right)$, solution of the first order differential equation

$$
\wp^{2}=4 \wp^{3}-g_{2} \wp-g_{3},
$$

such that the solutions become

$$
\left\{\begin{array}{l}
U=-6\left(\wp_{1}(\xi)+\wp_{2}(\xi)\right) \\
V=-6\left(\wp_{1}(\xi)-\wp_{2}(\xi)\right)
\end{array}\right.
$$

\section{Canonical transformation between SK and KK.}

Let us consider, for $\mu$ arbitrary, the equations and the constants of motion associated with the SK and KK Hamiltonians 14,15

$$
\mathrm{SK}: \quad U^{\prime}=P_{U}, \quad V^{\prime}=P_{V},
$$




$$
\begin{aligned}
& U^{\prime \prime}=-\frac{1}{2}\left(V^{2}+U^{2}\right)-\frac{c}{2}, \quad V^{\prime \prime}=-U V+\frac{\mu}{4 V^{3}}, \\
& K_{1}=\frac{1}{2}\left(P_{U}^{2}+P_{V}^{2}\right)+\frac{1}{2} U V^{2}+\frac{1}{6} U^{3}-\frac{c}{2} U+\frac{\mu}{8 V^{2}}, \\
& K_{2}^{2}=K_{2,0}^{2}+\frac{2}{3} \mu U+\mu \frac{P_{U}^{2}}{V^{2}}
\end{aligned}
$$

$\mathrm{KK}: \quad a=\frac{1}{4}, \quad c_{1}=16 c_{2}, \quad c=c_{1} c_{2}, \quad u=X+2 c_{2}, \quad v=Y$,

$$
u^{\prime}=p_{u}, \quad v^{\prime}=p_{v},
$$

$$
\begin{aligned}
& u^{\prime \prime}=-\frac{1}{4} v^{2}-4 u^{2}+c, \quad v^{\prime \prime}=-\frac{1}{2} u v+\frac{\mu}{v^{3}}, \\
& k_{1}=\frac{1}{2}\left(p_{u}^{2}+p_{v}^{2}\right)+\frac{1}{4} u v^{2}+\frac{4}{3} u^{3}-c u+\frac{1}{2} \frac{\mu}{v^{2}},
\end{aligned}
$$

$$
k_{2,0}^{2}=p_{v}^{4}-\frac{1}{72} v^{6}-\frac{1}{12} u^{2} v^{4}+u p_{v}^{2} v^{2}-\frac{1}{3} p_{u} p_{v} v^{3}+\frac{c}{12} v^{4},
$$

$k_{2}^{2}=k_{2,0}^{2}+\frac{\mu}{3} u+2 \mu \frac{p_{v}^{2}}{v^{2}}+\frac{\mu^{2}}{v^{4}}$.

The reason why the expressions $k_{2,0}$ and $k_{2}$ are defined by their square will appear soon.

The two nonlinear partial differential equations SK and KK

$$
\begin{array}{ll}
\text { SK: } & U_{t}+\left(U_{x x x x}+5 U U_{x x}+\frac{5}{3} U^{3}\right)_{x}=0, \\
\mathrm{KK}: & u_{t}+\left(u_{x x x x}+10 u u_{x x}+\frac{20}{3} u^{3}+30 u_{x}^{2}\right)_{x}=0,
\end{array}
$$

whose reductions $\xi=x-c t$ are (11) and (13), respectively obtained from the systems (25)-(26) and (30)-(31) by elimination of the variables $V$ and $v$, possess a Lax pair with a third order scattering problem $(L \psi=\lambda \psi) .16 .17$ The scattering operators can be factorized in the following way

$$
\begin{aligned}
\mathrm{SK}: & L \equiv \partial_{x}^{3}+U \partial_{x}=\left(\partial_{x}-w\right)\left(\partial_{x}+w\right) \partial_{x}, \\
\mathrm{KK}: & L \equiv \partial_{x}^{3}+2 u \partial_{x}+u_{x}=\left(\partial_{x}+w\right)\left(\partial_{x}\right)\left(\partial_{x}-w\right),
\end{aligned}
$$

such that the solutions of the PDE's are related through a Bäcklund transformation

$$
\begin{aligned}
\text { SK: } & U=w_{x}-w^{2} \\
\mathrm{KK}: & u=-w_{x}-\frac{1}{2} w^{2} .
\end{aligned}
$$


with the solution $w$ of the Fordy Gibbons equation 12

$$
w_{t}+\left(w_{4 x}-5 w_{x} w_{x x}-5 w^{2} w_{x x}-5 w w_{x}^{2}+w^{5}\right)_{x}=0 .
$$

The reduction $\xi=x-c t$ of this equation can be solved for $w(\xi)$, either by eliminating $U$ and $w^{\prime}$ between (38) and the equations of motion (25)

$$
w=\frac{1}{2} \frac{\sqrt{-\mu}}{V^{2}}-\frac{V^{\prime}}{V}
$$

or by eliminating $u$ and $w^{\prime}$ between (39) and the equations of motion (30)

$$
w=2 \frac{\sqrt{-\mu}}{v^{2}}+2 \frac{v^{\prime}}{v}
$$

such that, defining $\lambda^{2}=-\mu$ and

$$
\begin{aligned}
\Gamma= & 6\left(V K_{2,0}+\lambda P_{U}\right), \\
\Omega= & 48\left(3 v^{4} k_{2,0}^{2}+6 \lambda u v^{5} p_{v}+12 \lambda p_{v}^{3} v^{3}-\lambda v^{6} p_{u}\right. \\
& \left.+3 \lambda^{2} u v^{4}+18 \lambda^{2} v^{2} p_{v}^{2}+12 \lambda^{3} v p_{v}+3 \lambda^{4}\right),
\end{aligned}
$$

the canonical transformation is given by 11.18

$$
\begin{aligned}
u= & -\frac{3}{2}\left(-\frac{P_{V}}{V}+\frac{\lambda}{2 V^{2}}\right)^{2}-U, \quad v^{2}=\frac{\Gamma}{V^{2}}, \\
p_{u}= & \frac{1}{V^{3}}\left(3 P_{V}^{3}+3 U V^{2} P_{V}-P_{U} V^{3}\right) \\
& -\frac{3 \lambda}{2 V^{6}}\left(U V^{4}+3 V^{2} P_{V}^{2}-\frac{3}{2} \lambda V P_{V}+\frac{\lambda^{2}}{4}\right), \\
p_{v}= & \frac{1}{4 V^{2}}\left(-2 P_{V}+\frac{\lambda}{V}\right) \sqrt{\Gamma}-\lambda \frac{V}{\sqrt{\Gamma}}, \\
U= & -6\left(\frac{p_{v}}{v}+\frac{\lambda}{v^{2}}\right)^{2}-u, \quad V^{2}=\frac{\Omega}{4 v^{8}}, \\
P_{U}= & \frac{1}{v^{3}}\left(12 p_{v}^{3}+6 u v^{2} p_{v}-v^{3} p_{u}\right) \\
& +\frac{3 \lambda}{v^{6}}\left(2 u v^{4}+12 v^{2} p_{v}^{2}+12 \lambda v p_{v}+4 \lambda^{2}\right), \\
P_{V}= & -\frac{1}{v^{5}}\left(p_{v}+\frac{\lambda}{v}\right) \sqrt{\Omega}+\lambda \frac{v^{4}}{\sqrt{\Omega}} \cdot
\end{aligned}
$$




\section{General solution of the Kaup-Kupershmidt case}

Starting from the separation of variables $(17)$ and the canonical transformation (47)-(48) in the case $\mu=0$, we consider the transformation defined by 10 on the basis of Painlevé analysis

$$
\left\{\begin{array}{l}
q_{1}=-6 \frac{p_{v}^{2}-k_{2,0}}{v^{2}}-u \\
q_{2}=-6 \frac{p_{v}^{2}+k_{2,0}}{v^{2}}-u \\
p_{1}=\frac{1}{2 v^{3}}\left(12 p_{v}^{3}+6 u v^{2} p_{v}-v^{3} p_{u}-12 p_{v} k_{2,0}\right) \\
p_{2}=\frac{1}{2 v^{3}}\left(12 p_{v}^{3}+6 u v^{2} p_{v}-v^{3} p_{u}+12 p_{v} k_{2,0}\right)
\end{array}\right.
$$

This inverts to

$$
\left\{\begin{aligned}
u & =-6\left(\frac{p_{2}-p_{1}}{q_{2}-q_{1}}\right)^{2}-\frac{1}{2}\left(q_{1}+q_{2}\right) \\
v^{2} & =\frac{12 k_{2,0}}{q_{1}-q_{2}} \\
p_{u} & =24\left(\frac{p_{1}-p_{2}}{q_{1}-q_{2}}\right)^{3}+2\left(p_{1}-p_{2}\right) \frac{q_{1}+q_{2}}{q_{1}-q_{2}}+2 \frac{p_{1} q_{2}-p_{2} q_{1}}{q_{1}-q_{2}} \\
p_{v}^{2} & =12 k_{2,0} \frac{\left(p_{2}-p_{1}\right)^{2}}{\left(q_{1}-q_{2}\right)^{3}}
\end{aligned}\right.
$$

Taking account that $k_{2,0}$ is no more a constant of motion, this change of variables will appear be useful to find the general solution for KK. In those new variables, the Hamiltonian system (31) becomes

$$
\begin{aligned}
H & \equiv k_{1}=p_{1}^{2}+p_{2}^{2}+\frac{1}{12}\left(q_{1}^{3}+q_{2}^{3}\right)-\frac{c}{4}\left(q_{1}+q_{2}\right)+\frac{\mu}{24} \frac{q_{1}-q_{2}}{k_{2,0}}, \\
k_{2,0} & =2\left(p_{2}^{2}-p_{1}^{2}\right)+\frac{1}{6}\left(q_{2}^{3}-q_{1}^{3}\right)-\frac{c}{2}\left(q_{2}-q_{1}\right), \\
q_{1}^{\prime} & =2 p_{1}+\frac{\mu}{6} \frac{\left(q_{1}-q_{2}\right) p_{1}}{k_{2,0}^{2}}, \\
q_{2}^{\prime} & =2 p_{2}-\frac{\mu}{6} \frac{\left(q_{1}-q_{2}\right) p_{2}}{k_{2,0}^{2}} .
\end{aligned}
$$

Therefore, defining $f\left(q_{i}, p_{i}\right) \equiv p_{i}^{2}+\frac{1}{12} q_{i}^{3}-\frac{c}{4} q_{i} \quad(i=1,2)$, the HamiltonJacobi equation is separated

$$
k_{1}\left(f\left(q_{1}, p_{1}\right)-f\left(q_{2}, p_{2}\right)\right)=f^{2}\left(q_{1}, p_{1}\right)-f^{2}\left(q_{2}, p_{2}\right)+\frac{\mu}{48}\left(q_{1}-q_{2}\right),(55)
$$




$$
p_{i}=\frac{\partial S}{\partial q_{i}} \quad(i=1,2)
$$

We can express the second constant of motion $k_{2}^{2}$ in two equivalent ways

$$
\begin{aligned}
k_{2}^{2} & =-\frac{\mu}{3} q_{1}+\left(k_{2,0}+\frac{\mu}{12} \frac{q_{1}-q_{2}}{k_{2,0}}\right)^{2}, \\
\text { or } \quad k_{2}^{2} & =-\frac{\mu}{3} q_{2}+\left(k_{2,0}-\frac{\mu}{12} \frac{q_{1}-q_{2}}{k_{2,0}}\right)^{2},
\end{aligned}
$$

so that the elimination of $\mu\left(q_{1}-q_{2}\right) / k_{2,0}$ between (51), (56) and (51), (57) yields

$$
\begin{aligned}
k_{2}^{2} & =-\frac{\mu}{3} q_{1}+\left(-4 p_{1}^{2}-\frac{q_{1}^{3}}{3}+c q_{1}+2 k_{1}\right)^{2}, \\
\text { or } \quad k_{2}^{2} & =-\frac{\mu}{3} q_{2}+\left(4 p_{2}^{2}+\frac{q_{2}^{3}}{3}-c q_{2}-2 k_{1}\right)^{2} .
\end{aligned}
$$

The elimination of $p_{1}$ between (58) and (53), and of $p_{2}$ between (59) and (54) yields

$$
\begin{aligned}
& q_{1}^{\prime}=\sqrt{2 k_{1}-\frac{q_{1}^{3}}{3}+c q_{1}-\sqrt{k_{2}^{2}+\frac{\mu}{3} q_{1}}}\left(1+\frac{\mu}{3} \frac{q_{1}-q_{2}}{\left(\sqrt{k_{2}^{2}+\frac{\mu}{3} q_{2}}+\sqrt{k_{2}^{2}+\frac{\mu}{3} q_{1}}\right)^{2}}\right) \\
& q_{2}^{\prime}=\sqrt{2 k_{1}-\frac{q_{2}^{3}}{3}+c q_{2}+\sqrt{k_{2}^{2}+\frac{\mu}{3} q_{2}}}\left(1-\frac{\mu}{3} \frac{q_{1}-q_{2}}{\left(\sqrt{k_{2}^{2}+\frac{\mu}{3} q_{2}}+\sqrt{k_{2}^{2}+\frac{\mu}{3} q_{1}}\right)^{2}}\right) \cdot(
\end{aligned}
$$

In the case $\mu=0$, the differential equations for $q_{1}$ and $q_{2}$ are separated and their solution is expressed in terms of the Weierstrass elliptic function

$$
\begin{aligned}
& q_{1,0}=-12 \wp\left(\xi-\xi_{1}, \frac{c}{12},-\frac{1}{144}\left(2 k_{1,0}-k_{2,0}\right)\right) \equiv-12 \wp_{1}(\xi), \\
& q_{2,0}=-12 \wp\left(\xi-\xi_{2}, \frac{c}{12},-\frac{1}{144}\left(2 k_{1,0}+k_{2,0}\right)\right) \equiv-12 \wp_{2}(\xi),
\end{aligned}
$$

so that the solution for $(30)$ in the case $\mu=0$ is 10

$$
\begin{aligned}
& u=-\frac{3}{2}\left(\frac{\wp_{1}^{\prime}(\xi)-\wp_{2}^{\prime}(\xi)}{\wp_{1}(\xi)-\wp_{2}(\xi)}\right)^{2}+6\left(\wp_{1}(\xi)+\wp_{2}(\xi)\right), \\
& v^{2}=\frac{k_{2,0}}{\wp_{2}(\xi)-\wp_{1}(\xi)} .
\end{aligned}
$$


In the case $\mu \neq 0$, let us introduce the new variables

$$
s_{1}=\sqrt{3 \frac{k_{2}^{2}}{\mu}+q_{1}}, \quad s_{2}=-\sqrt{3 \frac{k_{2}^{2}}{\mu}+q_{2}},
$$

which transform the equations $(60)$ and (61) into

$$
\begin{aligned}
& s_{1}^{\prime}=\sqrt{2 k_{1}-\frac{1}{3}\left(s_{1}^{2}-3 \frac{k_{2}^{2}}{\mu}\right)^{3}+c\left(s_{1}^{2}-3 \frac{k_{2}^{2}}{\mu}\right)-\sqrt{\frac{\mu}{3}} s_{1}}\left(\frac{1}{s_{1}-s_{2}}\right) \\
& s_{2}^{\prime}=-\sqrt{2 k_{1}-\frac{1}{3}\left(s_{2}^{2}-3 \frac{k_{2}^{2}}{\mu}\right)^{3}+c\left(s_{2}^{2}-3 \frac{k_{2}^{2}}{\mu}\right)-\sqrt{\frac{\mu}{3}}} s_{2}\left(\frac{1}{s_{1}-s_{2}}\right) .
\end{aligned}
$$

Defining

$$
P(s)=2 k_{1}-\frac{1}{3}\left(s^{2}-3 \frac{k_{2}^{2}}{\mu}\right)^{3}+c\left(s^{2}-3 \frac{k_{2}^{2}}{\mu}\right)-\sqrt{\frac{\mu}{3}} s,
$$

the system (67)- 68) can be solved by inversion of the hyperelliptic integrals

$$
\begin{aligned}
& \int_{\infty}^{s_{1}} \frac{\mathrm{d} s}{\sqrt{P(s)}}+\int_{\infty}^{s_{2}} \frac{\mathrm{d} s}{\sqrt{P(s)}}=k_{3}, \\
& \int_{\infty}^{s_{1}} \frac{s \mathrm{~d} s}{\sqrt{P(s)}}+\int_{\infty}^{s_{2}} \frac{s \mathrm{~d} s}{\sqrt{P(s)}}=\xi+k_{4},
\end{aligned}
$$

which define $s_{1}$ and $s_{2}$ as multivalued functions of $\xi 1920$

The general solution of the equations of motion (30) in the case $\mu \neq 0$ is

$$
\begin{aligned}
& u=-\frac{1}{2}\left(s_{1}^{2}+s_{2}^{2}\right)+\frac{3}{\mu} k_{2}^{2}-\frac{3}{2}\left(\frac{s_{1}^{\prime}+s_{2}^{\prime}}{s_{1}+s_{2}}\right)^{2}, \\
& v^{2}=\frac{2 \sqrt{3 \mu}}{s_{1}+s_{2}} .
\end{aligned}
$$

As they are rational symmetric combinations of $s_{1}$ and $s_{2}$ and their derivatives, $u$ and $v^{2}$ are single-valued functions of $\xi$.

In the variables $q_{1}, q_{2}$ this solution is expressed as

$$
\begin{aligned}
u= & -\frac{3}{2}\left(\frac{\sqrt{2 k_{1}-\frac{q_{1}^{3}}{3}+c q_{1}-\sqrt{k_{2}^{2}+\frac{\mu}{3} q_{1}}}-\sqrt{2 k_{1}-\frac{q_{2}^{3}}{3}+c q_{2}+\sqrt{k_{2}^{2}+\frac{\mu}{3} q_{2}}}}{q_{1}-q_{2}}\right)^{2} \\
& -\frac{1}{2}\left(q_{1}+q_{2}\right), \\
v^{2}= & 6 \frac{\sqrt{k_{2}^{2}+\frac{\mu}{3} q_{1}}+\sqrt{k_{2}^{2}+\frac{\mu}{3} q_{2}}}{q_{1}-q_{2}},
\end{aligned}
$$


which clearly goes to (64)-(65) in the limit $\mu \rightarrow 0$.

\section{General solution of the Sawada-Kotera case}

We start from the general solution $(\sqrt{72})$, $(\sqrt{73})$ for KK and apply the canonical transformation (47) to obtain the general solution for the SK Hamiltonian system

$$
\begin{aligned}
U & =\sqrt{-3}\left(s_{1}^{\prime}+s_{2}^{\prime}\right)+s_{1}^{2}+s_{1} s_{2}+s_{2}^{2}-\frac{3}{\mu} K_{2}^{2} \\
V^{2} & =-2 \sqrt{-3}\left(s_{1}+s_{2}\right)\left(s_{1} s_{1}^{\prime}+s_{2} s_{2}^{\prime}\right)+2\left(s_{1}+s_{2}\right)^{2}\left(s_{1}^{2}+s_{2}^{2}-\frac{9 K_{2}^{2}}{2 \mu}\right) .
\end{aligned}
$$

Let us check that the limit of this solution when $\mu \rightarrow 0$ is (23). The expression (76) for $U$ can also be written as

$$
\begin{aligned}
U= & \frac{1}{2}\left(s_{1}^{2}+s_{2}^{2}\right)-\frac{3}{\mu} K_{2}^{2}+\frac{3}{2}\left(\frac{s_{1}^{\prime}+s_{2}^{\prime}}{s_{1}+s_{2}}\right)^{2} \\
& -\frac{3}{2}\left(\frac{s_{1}^{\prime}+s_{2}^{\prime}}{s_{1}+s_{2}}+\sqrt{-\frac{1}{3}}\left(s_{1}+s_{2}\right)\right)^{2} .
\end{aligned}
$$

Since in the limit $\mu \rightarrow 0$

$$
\begin{aligned}
& s_{1}+s_{2}=\mathcal{O}(\sqrt{\mu}), \\
& \frac{s_{1}^{\prime}+s_{2}^{\prime}}{s_{1}+s_{2}} \rightarrow \frac{\wp_{1}^{\prime}(\xi)-\wp_{2}^{\prime}(\xi)}{\wp_{1}(\xi)-\wp_{2}(\xi)},
\end{aligned}
$$

one has

$$
\lim _{\mu \rightarrow 0} U=\frac{1}{2}\left(Q_{1}+Q_{2}\right)
$$

Next, for $V^{2}$, the expansions

$$
\begin{gathered}
2\left(s_{1}+s_{2}\right)^{2}\left(s_{1}^{2}+s_{2}^{2}-\frac{9 K_{2}^{2}}{2 \mu}\right)=\frac{1}{4}\left(Q_{1}-Q_{2}\right)^{2}+\mathcal{O}(\mu), \mu \rightarrow 0, \\
\left(s_{1}+s_{2}\right)\left(s_{1} s_{1}^{\prime}+s_{2} s_{2}^{\prime}\right)=\mathcal{O}(\sqrt{\mu}), \mu \rightarrow 0,
\end{gathered}
$$

provide the limit of $V^{2}$ in (77). Therefore,

$$
\lim _{\mu \rightarrow 0} V^{2}=\frac{1}{4}\left(Q_{1}-Q_{2}\right)^{2} .
$$




\section{Comparison with the results of Cosgrove}

C. Cosgrove 13 recently integrated the ODE's (11) and (13) with hyperelliptic functions, using the postmultiplier method.

To compare the two different ways of integration, let us recall the vocabulary introduced in Painlevé analysis of nonlinear differential equations, making the distinction between fixed and movable constants. A constant is called fixed if it appears explicitly in the differential equation, while it is movable if it is a constant of integration and therefore depends on the initial data.

In the Hamiltonian formalism, described by the system (28)-(33) and (24)-(27), $k_{1}$ (resp. $K_{1}$ ) and $k_{2}$ (resp. $K_{2}$ ) are movable constants, while $\mu$ is fixed (it appears in the equations of motion).

In Cosgrove's paper, the first integrals of the fourth order equation he obtained in formulas (4.3)-(4.4) and (5.6)-(5.7), which are therefore movable constants, correspond in the Hamiltonian formalism to $k_{2}$ (resp. $K_{2}$ ) and $\mu$, respectively introduced as movable and fixed constants. In order to integrate the resulting second order sixth degree differential equation and transform it in a coupled system of first order equations for applying the postmultiplier method, Cosgrove defined "suitable" auxiliary variables chosen in a "subjective" way. Here the link between the canonical variables and its expressions (4.5)-(4.6) and (5.3)-(5.4) can be clearly established. Those expressions, which are "hidden" variables for the fourth order differential equation, are nothing else than the canonical variables $v^{2}$ (resp. $V^{2}$ ) and $p_{v}^{2}$ $\left(\right.$ resp. $P_{V}^{2}$ ) explicitly defined in the Hamiltonian formalism.

\section{Conclusion}

We have proven that the three integrable cases $\left(\mathrm{SK}, \mathrm{KdV}_{5}, \mathrm{KK}\right)$ of the Hénon-Heiles Hamiltonian can be integrated in terms of hyperelliptic functions.

We will take advantage of the method developed here to integrate other Hamiltonian systems with two degrees of freedom and additional nonpolynomial terms.

\section{Acknowledgements}

The authors thank Vadim Kuznetsov and Pol Vanhaecke for interest-

ing and useful discussions. They acknowledge the financial support of the 
Tournesol grant T99/040. MM and CV thank the Belgian government for the financial support extended within the framework of the IUAP contract $\mathrm{P} 4 / 08$. CV is a research assistant of the Fund for Scientific Research, Flanders. 


\section{References}

${ }^{1}$ T. Bountis, H. Segur, and F. Vivaldi, Phys. Rev. A 25, 1257 (1982).

${ }^{2}$ Y. F. Chang, M. Tabor, and J. Weiss, J. Math. Phys. 23, 531 (1982).

${ }^{3}$ B. Grammaticos, B. Dorizzi, and R. Padjen, Phys. Lett. A 89, 111 (1982).

${ }^{4}$ A. P. Fordy, Phys. D 52, 204 (1991).

${ }^{5}$ J. Drach, C. R. Acad. Sc. Paris 168, 337 (1919).

${ }^{6}$ A. Ankiewicz and C. Pask, J. Phys. A 16, 4203 (1983).

${ }^{7}$ S. Wojciechowski, Phys. Lett. A 100, 277 (1984).

${ }^{8}$ J. Chazy, Thèse, Paris (1910); Acta Math. 34, 317 (1911).

${ }^{9}$ Y. Aizawa and N. Saitô, J. Phys. Soc. Jpn 32, 1636 (1972).

${ }^{10}$ V. Ravoson, L. Gavrilov, and R. Caboz, J. Math. Phys. 34, 2385 (1993).

${ }^{11}$ S. Baker, PhD Thesis, University of Leeds (1995).

${ }^{12}$ A. P. Fordy and J. Gibbons, J. Math. Phys. 21, 2508 (1980).

${ }^{13}$ C. M. Cosgrove, Studies in Appl. Math. 104, 1 (2000).

${ }^{14}$ B. Grammaticos, B. Dorizzi, and A. Ramani, J. Math.Phys. 25, 3470 (1984).

${ }^{15}$ J. Hietarinta, Phys. Rep. 147, 87 (1987).

${ }^{16}$ J. Satsuma and D. J. Kaup, J. Phys. Soc. Jpn 43, 692 (1977).

${ }^{17}$ D. J. Kaup, Stud. Appl. Math. 62, 186 (1980).

${ }^{18}$ M. Błaszak and S. Rauch-Wojciechowski, J. Math. Phys. 35, 1693 (1994).

${ }^{19}$ Sophie Kowalevski, Acta Math. 12, 177 (1889).

${ }^{20} \mathrm{~V}$. V. Golubev, Lectures on the integration of the equation of motion of a rigid body about a fixed point, Gostechizdat (State publishing house), Moscow, 1953. English: Israel program for scientific translations, 1960. 